\title{
Homogeneous Graft Copolymerization of Vinyl Monomers onto Cellulose in a Dimethyl Sulfoxide-Paraformaldehyde Solvent System IV. 2-Hydroxyethyl Methacrylate
}

\author{
Noboru NishioKa, Yoshiyuki Matsumoto, Toshiyuki Yumen, \\ Kouji MONMAE, and Kouichi KOSAI \\ Faculty of Engineering, Osaka Electro-Communication University, \\ Neyagawa, Osaka 572, Japan
}

(Received March 22, 1985)

\begin{abstract}
The homogeneous graft copolymerization of 2-hydroxyethyl methacrylate (HEMA) onto cellulose was carried out under various conditions. Ammonium persulfate (APS), potassium persulfate (KPS), and azobisisobutyronitrile (AIBN) were used as radical initiators. The optimum grafting condition, the molecular weight of graft polymer, and the number of grafts per cellulose chain for each initiator system were determined as functions of polymerization temperature and concentration of monomer, initiator, and cellulose. Grafting onto cellulose proceeded in the APS and KPS systems, but hardly in the AIBN system. The molecular weight of graft polymer increased with increasing monomer and cellulose concentration but decreased with increasing temperature and initiator concentration. The number of grafts was found to be 5 and 0.2 at most for the APS and AIBN systems, respectively. KPS was found to be unsuitable as a grafting initiator owing to the degradation of the cellulose chains.
\end{abstract}

KEY WORDS Homogeneous Grafting / Cellulose / 2-Hydroxyethyl Methacrylate / Dimethyl Sulfoxide-Paraformaldehyde. Solvent System / Molecular Weight of Graft Polymer / Number of Grafts / Ammonium Persulfate / Potassium Persulfate / Azobisisobutyronitrile /

In our previous papers, ${ }^{1-3}$ the homogeneous graft copolymerization of vinyl monomers, i.e., acrylonitrile (AN), methyl methacrylate (MMA), and methyl acrylate (MA), onto cellulose in a dimethyl sulfoxide-paraformaldehyde (DMSO-PF) solvent system was carried out under various conditions to determine the optimum conditions for each monomer system. Characterization of the graft copolymers obtained was also carried out to elucidate the relationship between the grafting conditions and the structure of the graft copolymers.

The present study deals with the grafting of 2-hydroxyethyl methacrylate (HEMA) onto cellulose in the DMSO-PF solvent system. Ammonium persulfate (APS), potassium persulfate (KPS), and azobisisobutyronitrile
(AIBN) were tested as radical initiators. The optimum conditions under which grafting onto cellulose occurs most effectively were determined by varying the temperature and concentration of the initiator, monomer, and cellulose. Characterization of the graft copolymers obtained under various conditions was also carried out to estimate the molecular weights of the graft polymers and the number of grafts per cellulose chain.

Cellulose grafted with vinyl monomers in the DMSO-PF solvent system has been used as material for permselective membranes. ${ }^{4.5}$ Diffusive permeability of water soluble solutes through grafted cellulose membranes was found to depend remarkably on the state of water in them. ${ }^{6}$ It is known that HEMA is an amphiphilic monomer because of its $\alpha$-methyl 


\section{N. NishioKa et al.}

and hydroxy groups. ${ }^{7}$ Thus, the states of water in HEMA-grafted cellulose membranes differ from those in hydrophobic monomer grafted ones. Furthermore, cellulose grafted with HEMA will be suitable as hemodialysis membrane material because of the good biocompatibility of poly(2-hydroxyethyl methacrylate) (PHEMA). ${ }^{7}$

\section{EXPERIMENTAL}

Bleached softwood sulfite pulp was used as the cellulosic material. Practical grade PF dried in a desiccator over $\mathrm{CaCl}_{2}$ and reagent grade DMSO were used without further purification. The monomer was fractionally distilled under reduced pressure in a nitrogen atmosphere; $56^{\circ} \mathrm{C}(2 \mathrm{mmHg})$.

Thoroughly dried cellulose was dissolved in DMSO containing PF by a method described previously. ${ }^{1}$ The clear solution obtained, designated as the DMSO solution, was used as the stock cellulose solution. The homogeneous grafting of HEMA onto cellulose was carried out by a method described in our previous papers. ${ }^{1.3}$ The polymerization mixture was poured into a large amount of acetone with vigorous stirring to precipitate the reaction product. The crude graft product was treated with methanol in a Soxhlet apparatus for more than $48 \mathrm{~h}$ to extract the attendant homopolymer.

Some of the graft copolymers were hydrolyzed to isolate the graft polymers. A given sample was immersed in $72 \% \mathrm{H}_{2} \mathrm{SO}_{4}$ at room temperature for $6 \mathrm{~h}$. The reaction mixture was poured into acetone with vigorous stirring, and then the precipitate was collected by filtration. The precipitate was washed repeatedly with acetone, dried, and dissolved in methanol. After filtration, the graft PHEMA was recovered from the filtrate by precipitating into diethyl ether.

The intrinsic viscosity, $[\eta]$ (in $\mathrm{cm}^{3} \mathrm{~g}^{-1}$ ), of PHEMA in dimethylformamide (DMF) at $25^{\circ} \mathrm{C}$ was determined by an Ubbelohde type capillary viscometer. The following equation ${ }^{8}$ was used to estimate the molecular weight, $\bar{M}_{\mathrm{g}}$.

$$
[\eta]_{\mathrm{DMF}}^{25^{\circ} \mathrm{C}}=8.90 \times 10^{-5} M^{0.72}
$$

The molecular weight of backbone cellulose was estimated to be $16 \times 10^{4}$ from $[\eta]$ obtained with DMSO at $30^{\circ} \mathrm{C}^{9}$ and with cadoxen at $20^{\circ} \mathrm{C}^{10}$

The reaction product was characterized by the following parameters:

Weight conversion, $\mathrm{WC}(\%)$

$$
=\frac{\text { Total weight of PHEMA formed }}{\text { Weight of HEMA charged }} \times 100
$$

$$
\begin{aligned}
& \text { Grafting percentage, GP }(\%) \\
& =\frac{\text { Weight of graft PHEMA }}{\text { Weight of cellulose }} \times 100
\end{aligned}
$$

Grafting efficiency, GE (\%)

$$
=\frac{\text { Weight of graft PHEMA }}{\text { Total weight of PHEMA formed }} \times 100
$$

Number of gräts per cellulose chain, $\bar{N}_{\mathrm{g}}$

$$
\begin{gathered}
=\frac{\text { Molecular weighr of cellulose }}{\text { Molecular weight of graft PHEMA }} \\
\times \frac{\mathrm{GP}}{100}=\frac{16 \times 10^{4}}{\bar{M}_{\mathrm{g}}} \times \frac{\mathrm{GP}}{100}
\end{gathered}
$$

\section{RESULTS AND DISCUSSION}

Characterization of graft copolymers is necessary to elucidate their properties and grafting mechanisms. The first step for characterization of graft products is the separation of attendant homopolymers from crude graft products. The difficulty in separating attendant homopolymers from crude graft products has been pointed out by many workers. ${ }^{11-13}$ Thin layer chromatography (TLC) was found to be an effective technique for confirming the separation of attendant homopolymers. ${ }^{14-17}$ Quantitative analysis of chromatograms, 
however, is very troublesome. Chromatograms were analyzed only qualitatively in our previous papers, ${ }^{1-3}$ since Soxhlet extraction with a proper solvent was effective for the separation of the attendant homopolymers obtained in the DMSO-PF solvent system. This is probably because of the large solubility difference between cellulose and attendant homopolymers. ${ }^{18,19}$ Some of the graft products subjected to extraction were analyzed by TLC to confirm the separation of the PHEMA homopolymer.

Cellulose is so easily hydrolyzed to low molecular residues that graft polymers can be isolated. $^{18-20}$ It has been reported that PHEMA is resistent to hydrolysis because of its $\alpha$-methyl groups. ${ }^{21}$ The isolation of graft PHEMA was thus carried out with $\mathrm{H}_{2} \mathrm{SO}_{4}$ of various concentrations from 65 to $90 \%$. The residues hydrolyzed showed the characteristics of PHEMA in the infrared spectra but were not soluble completely in both methanol and DMF. Thus, only the soluble part in methanol was recovered to use for viscosity measurement. The residue hydrolyzed with $72 \%$ $\mathrm{H}_{2} \mathrm{SO}_{4}$ was obtained in the highest yield and those hydrolyzed with higher $\mathrm{H}_{2} \mathrm{SO}_{4}$ concentration were dark brown.

It is generally accepted that, in homogeneous grafting, the molecular weight of graft polymers is similar to that of attendant homopolymers. ${ }^{18-20}$ Moreover, these molecular weights were equal to each other within experimental error in the homogeneous grafting of AN and MMA onto cellulose in the DMSOPF solvent system. ${ }^{1,2}$ In this study, the molecular weights were also equal to each other; thus, the molecular weight of extracted attendant PHEMA was also used as that of graft PHEMA without any distinction.

It has been reported that the formation of free radicals by radiation and redox systems initiates the oxidative degradation of cellulose chains in the absence of monomer. ${ }^{22}$ In the presence of a monomer, however, one would expect cellulose degradation to be eliminated or at least minimized. ${ }^{20}$ Therefore, the molecular weight of cellulose determined before grafting was used as that of cellulose in the graft copolymer under the assumption that the degradation of cellulose is negligible during grafting. This assumption is confirmed in Figure 6 discussed below.

\section{Initiation with Ammonium Persulfate}

Two peroxide initiators were tested for the grafting of HEMA onto cellulose. The results obtained with APS are discussed first.

Grafting was carried out at different fixed temperatures ranging from 20 to $80^{\circ} \mathrm{C}$ while the concentrations of HEMA and APS and the polymerization time were kept constant. As can be seen from Figure 1a, the weight conversion and grafting percentage show maxima around $40^{\circ} \mathrm{C}$, as is also the case for the grafting of AN, MMA, and MA onto cellulose in the DMSO-PF solvent system. ${ }^{1-3}$ On the other hand, the grafting efficiency decreases gradually with increasing temperature. A similar temperature dependence of grafting efficiency has been found in our previous studies in this solvent system $^{1-3}$ and other grafting systems, ${ }^{23}$ implying that the activation energy of the propagation of PHEMA is larger than that of the formation of cellulose macroradicals.

Figure $1 \mathrm{~b}$ shows the temperature dependence of the molecular weight of graft PHEMA and the number of grafts per cellulose chain. The molecular weight of graft PHEMA decreases with increasing temperature but the number of grafts increases gradually; this is consistent with expectation based on the kinetics of radical polymerization. ${ }^{19}$ The grafting percentage is proportional to the product of the molecular weight of graft PHEMA and the number of grafts. The decrease in grafting percentage at higher temperatures thus indicates that the decrease in the molecular weight of graft PHEMA is more pronounced than the increase in the number of grafts.

Figure 2 shows the effect of APS con- 


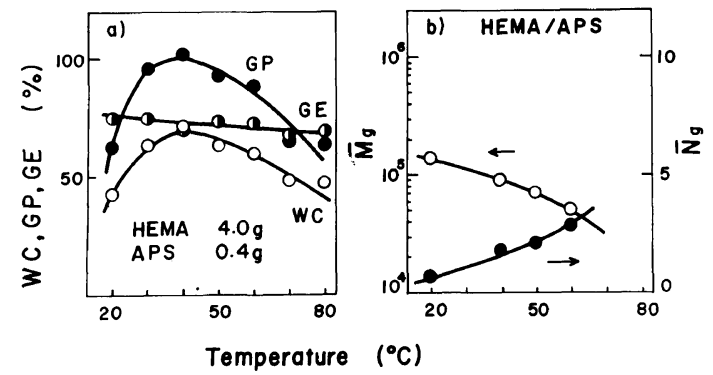

Figure 1. Effects of polymerization temperature on the grafting of HEMA with APS: (a) (O) weight conversion, WC; (O) grafting percentage, GP; (O) grafting efficiency, GE; (b) (O) molecular weight of graft PHEMA, $\bar{M}_{\mathrm{g}} ;$ (О) number of grafts per cellulose chain, $\bar{N}_{\mathrm{g}}$; grafting time, $5 \mathrm{~h}$; cellulose concn, $2 \%$; HEMA concn, $4.0 \mathrm{~g} / 100 \mathrm{~g}$ DMSO soln; APS concn, $0.4 \mathrm{~g} / 100 \mathrm{~g}$ DMSO soln.

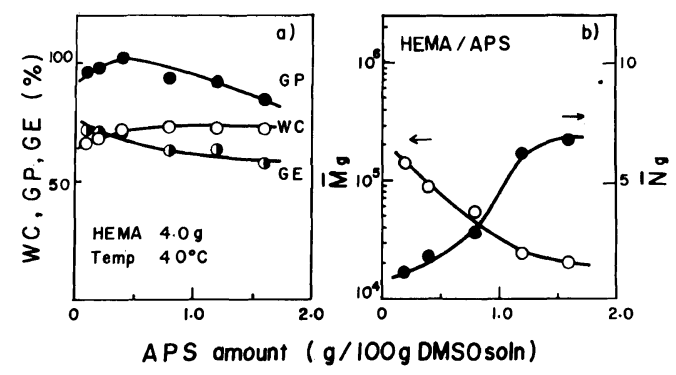

Figure 2. Effects of initiator concentration of the grafting of HEMA with APS. The symbols are the same as those in Figure 1. Grafting time, $5 \mathrm{~h}$; cellulose concn, $2 \%$; polymerization temp, $40^{\circ} \mathrm{C}$.

centration. The initiator concentration is expressed in grams of APS in $100 \mathrm{~g}$ of the DMSO solution and all experiments were carried out at $40^{\circ} \mathrm{C}$ which is, as can be seen from Figure 1, the optimum condition with respect to weight conversion and grafting percentage. The weight conversion is nearly constant irrespective of the increase in APS concentration and levels off at about $70 \%$, whereas the grafting percentage shows a broad maximum around $0.4 \mathrm{~g}$. On the other hand, the grafting efficiency decreases gradually with increasing APS concentration; this is consistent with expectation based on the kinetics of radical polymerization. ${ }^{19}$

An increase in initiator concentration prob-

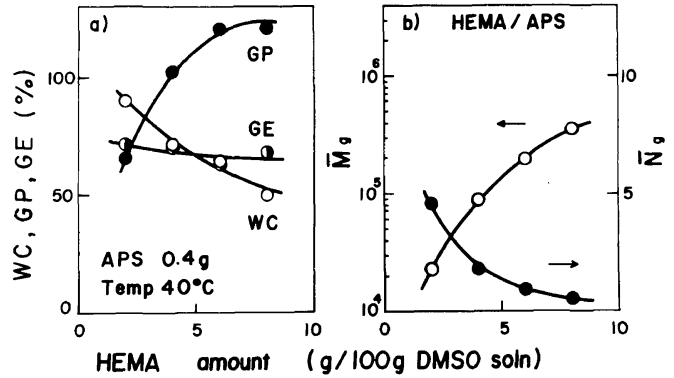

Figure 3. Effects of monomer concentration on the grafting of HEMA with APS. The symbols are the same as those in Figure 1. Grafting time, $5 \mathrm{~h}$; cellulose concn, $2 \%$.

ably causes both an increase in the number of grafts and a decrease in the molecular weight of graft polymer. The results in Figure $2 b$ are consistent with this expectation. The leveling off in the number of grafts at higher APS concentrations is ascribed to the saturation of radical concentration, since a further increase in APS concentration would lead to termination of the primary radicals. The decrease in grafting percentage at higher APS concentrations is attributable to the decrease in the molecular weight of the graft polymer.

Figure 3 shows the effects of monomer concentration expressed in grams of HEMA in $100 \mathrm{~g}$ of the DMSO solution. The initiator concentration was kept at $0.4 \mathrm{~g}$ which is, as seen from Figure 2, the optimum condition with respect to the grafting percentage. The weight conversion decreases with increasing HEMA concentration, though the total amount of HEMA polymerized increases gradually. Such a trend is comparable with our previous results; ${ }^{1,3}$ the weight conversion increased slightly with the increase in monomer concentration. The addition of monomer, a nonsolvent for cellulose, to the DMSO solution resulted in gelation. In the case of HEMA, this trend was remarkable. Reproducible data were not obtained at higher HEMA concentrations than $10 \mathrm{~g}$ because of gelation during grafting.

On the other hand, the grafting percentage 


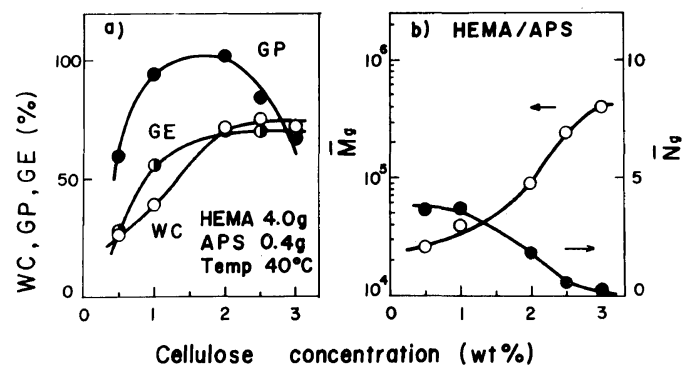

Figure 4. Effects of cellulose concentration on the grafting of HEMA with APS. The symbols are the same as those in Figure 1. Grafting time, $5 \mathrm{~h}$.

increased with increasing HEMA concentration and leveled off at about $120 \%$, whereas the grafting efficiency decreased gradually, indicating that homopolymerization becomes predominant at higher HEMA concentrations. An increase in monome concentration facilitates the consumption of primary radicals, thus reducing the formation of cellulose macroradicals and decreasing the grafting efficiency and the number of grafts.

The effects of cellulose concentration is shown in Figure 4. The concentration of cellulose could not be changed over a wide range because of the high viscosity of the DMSO solution. The weight conversion and grafting efficiency increased with increasing cellulose concentration and leveld off, while the grafting percentage showed a maximum around $2 \%$. On the other hand, the molecular weight increased with increasing cellulose concentration but the number of grafts decreased.

An increase in cellulose concentration would facilitate the formation of cellulose macroradicals because of an increase in the relative ratio of cellulose to primary radicals, leading to an increase in grafting efficiency. On the other hand, an increase in solution viscosity would lead to the gel effect, causing an increase in the molecular weight of graft polymers. The results in Figure 4 are consistent with these expectation. The decrease in grafting percentage at higher cellulose concentrations is attributed to the decrease in the

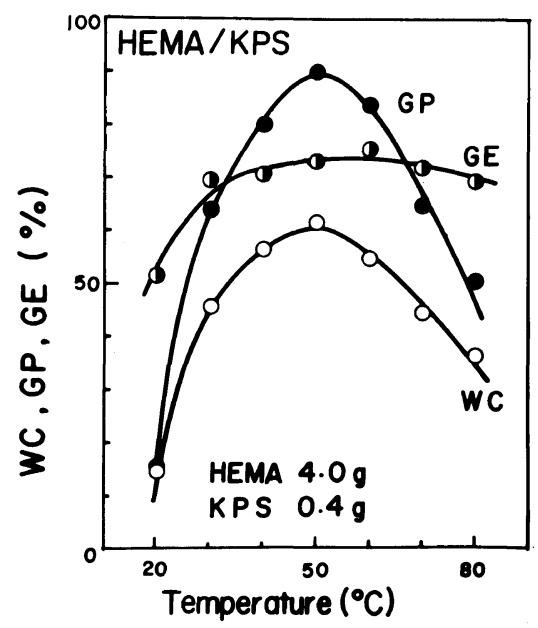

Figure 5. Effects of polymerization temperature on the grafting of HEMA with KPS. The symbols are the same as those in Figure 1. Grafting time, $5 \mathrm{~h}$; cellulose concn, $2 \%$.

number of grafts.

In the HEMA-APS system, the values of grafting percentage exceeded $100 \%$, being higher than those obtained in the previous studies. $^{1-3}$ This is due to the higher values of the molecular weight of graft PHEMA since the numbers of grafts for each monomer system is essentially the same.

\section{Initiation with Potassium Persulfate}

KPS was also used as an initiator for the grafting of HEMA onto cellulose. Figure 5 shows the effects of polymerization temperature. The weight conversion and grafting percentage show maxima around $50^{\circ} \mathrm{C}$. These results are similar to those shown in Figure 1a, whereas, in this initiator system, the solution viscosity decreases in the course of grafting. The decrease in solution viscosity was especially remarkable at higher temperatures and at higher initiator concentrations, implying the possibility of the degradation of cellulose chains. Such a decrease in solution viscosity in the course of grafting has also been reported when benzoyl peroxide (BPO) was used as an initiator in this solvent system. ${ }^{3}$ 


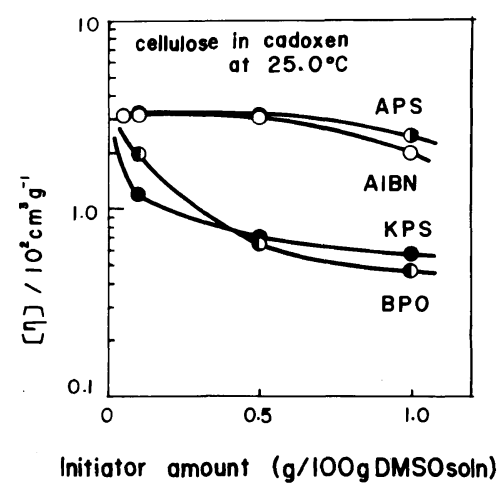

Figure 6. Initiator concentration dependence of intrinsic viscosity of cellulose in cadoxen at $25^{\circ} \mathrm{C}$. Cellulose was recovered from the DMSO soln containing initiator but without HEMA after stirring for $5 \mathrm{~h}$ at a given temperature. (O) APS at $40^{\circ} \mathrm{C}$; (O) $\mathrm{KPS}$ at $50^{\circ} \mathrm{C}$; (O) AIBN at $60^{\circ} \mathrm{C}$; (D) BPO at $60^{\circ} \mathrm{C}$.

The DMSO solution containing initiator but without HEMA was stirred for $5 \mathrm{~h}$ at a given temperature. The optimum temperature for each initiator system was for APS at $40^{\circ} \mathrm{C}$, $\mathrm{KPS}$ at $50^{\circ} \mathrm{C}$, AIBN at $60^{\circ} \mathrm{C}$, and $\mathrm{BPO}$ at $60^{\circ} \mathrm{C}$, respectively. The solution was then poured into distilled water to collect cellulose. The effects of initiator concentration on the intrinsic viscosity of recovered cellulose in cadoxen at $25^{\circ} \mathrm{C}$ is shown in Figure 6. The intrinsic viscosities of cellulose obtained in the APS and AIBN systems hardly changed with the increase in initiator concentration. Comparison of Figure 6 with Figure 2 discussed above and Figure 8 discussed below indicates that degradation of cellulose chains hardly takes place around the optimum conditions for both initiator systems.

On the other hand, the viscosities for the KPS and BPO systems decrease sharply at lower concentrations and gradually at higher concentrations, indicating the degradation of cellulose chains in these initiator systems and thus unsuitability as grafting initiators.

It is interesting to note that there exists no difference in the extent of cellulose degradation in the APS and AIBN systems. Furthermore, both APS and KPS are per- sulfate salts but there exists a difference in degradation. It has been reported that the decomposition rate of persulfate is greatly enhanced by the presence of poly(vinyl alcohol) (PVA) because of the redox reaction between PVA and persulfate ion ${ }^{24,25}$ and that the solution viscosity of PVA decreases remarkably with increasing reaction time in the APS system but hardly decreases in the AIBN system. ${ }^{26}$ The decomposition rate of persulfate was determined by iodometric titration. The enhancement of the decomposition rate of persulfate was recognized slightly in the presence of cellulose. But the decomposition rate of APS hardly differed from that of KPS. In the presence of an amine, e.g., benzylamine or ethylenediamine, cellulose chains also degraded easily in the DMSO-PF solvent system. ${ }^{27}$ The acidity or basicity of the DMSO solution may be related to the stability of cellulose chains. At present, the influence of persulfate on the degradation of cellulose chains remains unclear.

\section{Initiation with Azobisisobutyronitrile}

It is known that AIBN has an initiator effect arising from the resonance stabilization of its radical fragments, being not suitable for a grafting initiator. ${ }^{19}$ Homogeneous grafting onto cellulose in the DMSO-PF solvent system, however, indicated AIBN to be effective for the grafting of MMA but not for that of AN and MA. ${ }^{-3}$ Furthermore, the effectiveness of AIBN has been reported for other grafting systems. ${ }^{28-33}$ Thus, the grafting of HEMA in the DMSO-PF solvent system was also carried out with AIBN as an initiator.

The effect of temperature is shown in Figure 7. The weight conversion and grafting percentage show maxima around $50^{\circ} \mathrm{C}$, but the grafting efficiency is very low. The molecular weight of graft PHEMA decreases gradually with increasing temperature, which is consistent with the temperature effect of the APSHEMA system. The number of grafts is 0.2 at most. 


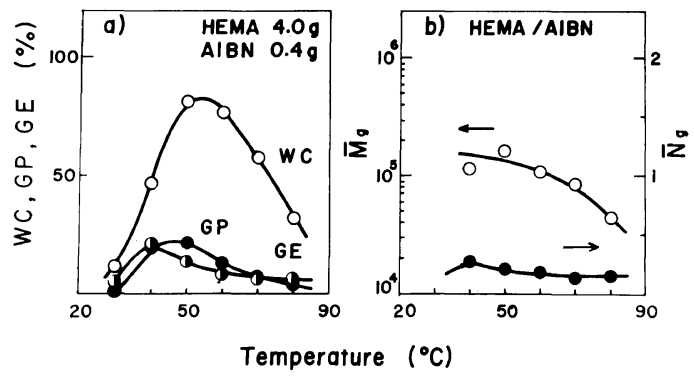

Figure 7. Effects of polymerization temperature on the grafting of HEMA with AIBN. The symbols are the same as those in Figure 1. Grafting time, $5 \mathrm{~h}$; cellulose concn, $2 \%$.

Figure 8 shows the effects of AIBN concentration. The weight conversion, grafting percentage, and grafting efficiency scarcely depend on AIBN concentration. On the other hand, the molecular weight of graft PHEMA decreases with increasing AIBN concentration, whereas the number of grafts increases to about one at higher concentration. Since the chain transfer from AIBN primary radicals to cellulose may be neglected due to resonance stabilization, these results indicate that the chain transfer from PHEMA homopolymer radicals to cellulose chains takes place slightly in this monomer-initiator system. Comparison of these results with those indicated in our previous studies shows the chain transfer reactivity of PHEMA macroradicals to cellulose to be higher than those of PAN and PMA macroradicals but not so high as that of PMMA macroradicals. ${ }^{1-3}$

\section{REFERENCES}

1. N. Nishioka and K. Kosai, Polym. J., 13, 1125 (1981).

2. N. Nishioka, K. Matsumoto, and K. Kosai, Polym. J., 15, 153 (1983).

3. N. Nishioka, K. Minami, and K. Kosai, Polym. J., 15, 591 (1983).

4. N. Nishioka, K. Watase, K. Arimura, K. Kosai, and M. Uno, Polym. J., 16, 867 (1984).

5. N. Nishioka, K. Kosai, and M. Uno, Polym. Prepr. Jpn., 33, 1699 (1984).

6. N. Nishioka, S. Yoshimi, T. Iwaguchi, and K. Kosai,

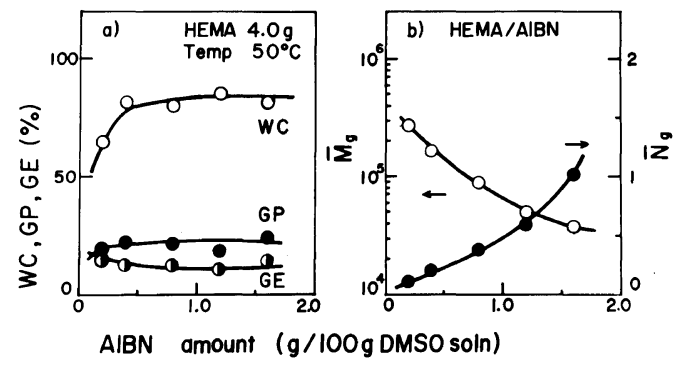

Figure 8. Effects of initiator concentration on the grafting of HEMA with AIBN. The symbols are the same as those in Figure 1. Grafting time, $5 \mathrm{~h}$; cellulose concn, $2 \%$.

Polym. J., 16, 877 (1984).

7. B. D. Ratner and A. S. Hoffman, "Synthetic Hydrogels for Biomedical Applications," J. D. Andrade, Ed., The American Chemical Society, Symposium Series, No. 31,1976.

8. M. Bohdanecky, Z. Tuzar, M. Stoll, and R. Chromecek, Collect. Czech., Chem. Commun., 33, 4104 (1968).

9. H. A. Swenson, Appl. Polym. Symp., 28, 945 (1976).

10. G. Jayme and P. Kleppe, Papier, 15, 492 (1961).

11. H. Yasuda, J. A. Wrag, and V. T. Stannett, J. Polym. Sci., C, 2, 387 (1963).

12. H. A. Ende and V. T. Stannett, J. Polym. Sci., A, 2, 4047 (1964).

13. I. Sakurada, Y. Ikada, and Y. Nishizaki, J. Polym. Sci., C, 37, 265 (1972).

14. T. Taga and H. Inagaki, Angew. Makromol. Chem., 33, 129 (1973).

15. H. Inagaki, T. Kotaka, and T. I. Min, Pure Appl. Chem., 46, 61 (1976).

16. T. I. Min and H. Inagaki, Polymer, 21, 309 (1980).

17. M. Tsuzuki, I. Hagiwara, N. Shiraishi, and T. Yokota, J. Appl. Polym. Sci., 25, 2909 (1980).

18. V. T. Stannett and H. B. Hopfenberg, "Cellulose and Cellulose Derivatives," Vol. V, Part V, N. M. Bikales and L. Segal, Ed., Wiley, New York, 1971, p 907.

19. F. Ide, "Graft Jugo to Sono Oyo," Kobunshi Kankokai, Kyoto, 1977.

20. A. Hebeish and J. T. Guthrie, "The Chemistry and Technology of Cellulosic Copolymers," SpringerVerlag, Berlin-Heiderberg-New York, 1981.

21. T. A. Jadwin, A. S. Hoffman, and W. R. Vieth, $J$. Appl. Polym. Sci., 14, 1339 (1970).

22. J. C. Arthur, Jr., J. Macromol. Sci., Chem., A4, 1057 (1970).

23. T. Maekawa, K. Hayashi, K. Uno, and S. Okamura, Kobunshi Kagaku, 21, 323 (1964).

24. F. Ide, K. Nakatsuka, and H. Tamura, Kobunshi Kagaku, 23, 45 (1966). 


\section{N. NisHIOKA et al.}

25. Y. Ikada, Y. Nishizaki, and I. Sakurada, J. Polym. Sci., Polym. Chem. Ed., 12, 1829 (1974).

26. S. Okamura and T. Yamashita, Kobunshi Kagaku, 15, 165 (1958).

27. K. Kosai and N. Nishioka, unpublished data.

28. K. Kosai, T. Higashino, and S. Ohue, Nippon Kagaku Kaishi, 523 (1975)

29. K. Kosai, T. Higashino, and N. Nishioka, Nippon Kagaku Kaishi, 561 (1977).
30. B. N. Misra, J. K. Jassal, and C. P. Pande, J. Polym. Sci., Polym. Chem. Ed., 16, 295 (1978).

31. B. N. Misra, R. Dogra, I. Kaur, and D. Scood, J. Polym. Sci., Polym. Chem. Ed., 18, 341 (1980).

32. B. N. Misra and R. Dogra, J. Macromol. Sci., Chem., A14, 763 (1980).

33. S. H. Egboh, M. H. George, J. A. Barrie, and D. J. Walsh, Makromol. Chem., 184, 725 (1983). 